\title{
Drawing Canyon: Sfumato presences in drawing and landscape
}

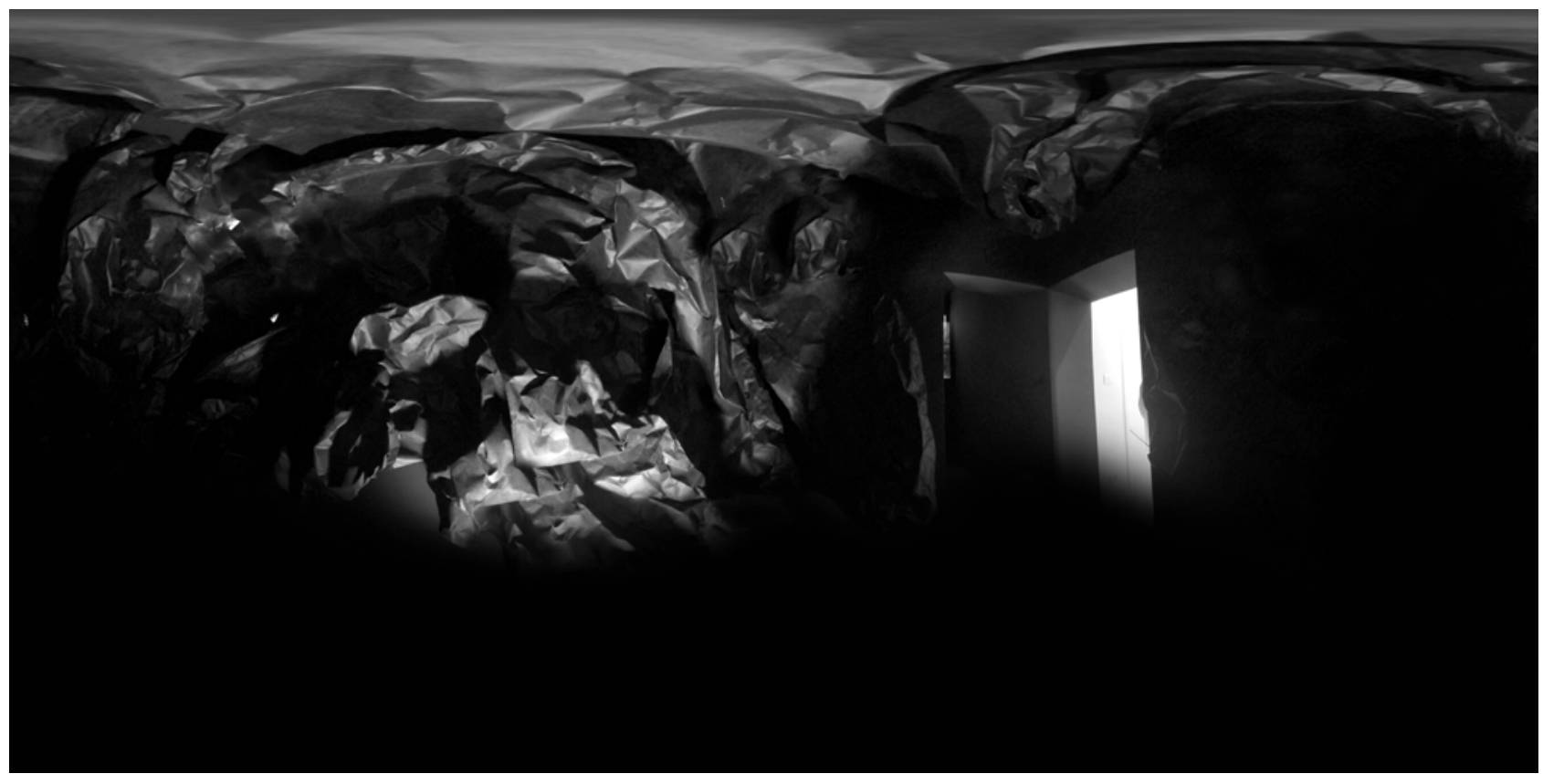

Fig. 1 Simon Twose (2018). South Bay, Kaikoura. The canyon is beneath the sea surface, beyond the reef [Photograph]

\section{Kaikōura canyon}

On a small vessel off the Kaikoura coast of Aotearoa/New Zealand, the landscape beneath the sea's surface is strangely present. The swells are at ocean scale, vast slow masses passing under the boat. Just five hundred metres from the shore the sea noticeably darkens above a line of undersea cliffs. These plummet vertically more than a kilometre into the Kaikoura canyon, which continues to deepen as it flows into the Hikurangi abyssal river, formed by the subduction of tectonic plates. Huge forces in this submarine landscape were released, two minutes after midnight on 14 November 2016, in the 7.8 magnitude Kaikoura earthquake; the seabed lurched upwards, triggering submarine rock falls, turbid flows of sediment, gas eruptions and tsunami. The hidden bathymetric landscape became suddenly present, violently sketching its latency in rock and water (Fig. 1, 2).

Canyon is a multi-media drawing experiment that projects into the imagined space of the Kaikoura canyon. We imagine the presences in the submarine material/ space in concert with crude, hand drawn sketches that are intensified through virtual reality (VR) and spatialised sound. The blurs and smudges in these hybrid sketches that contribute to their openness and indeterminacy, are paralleled with 


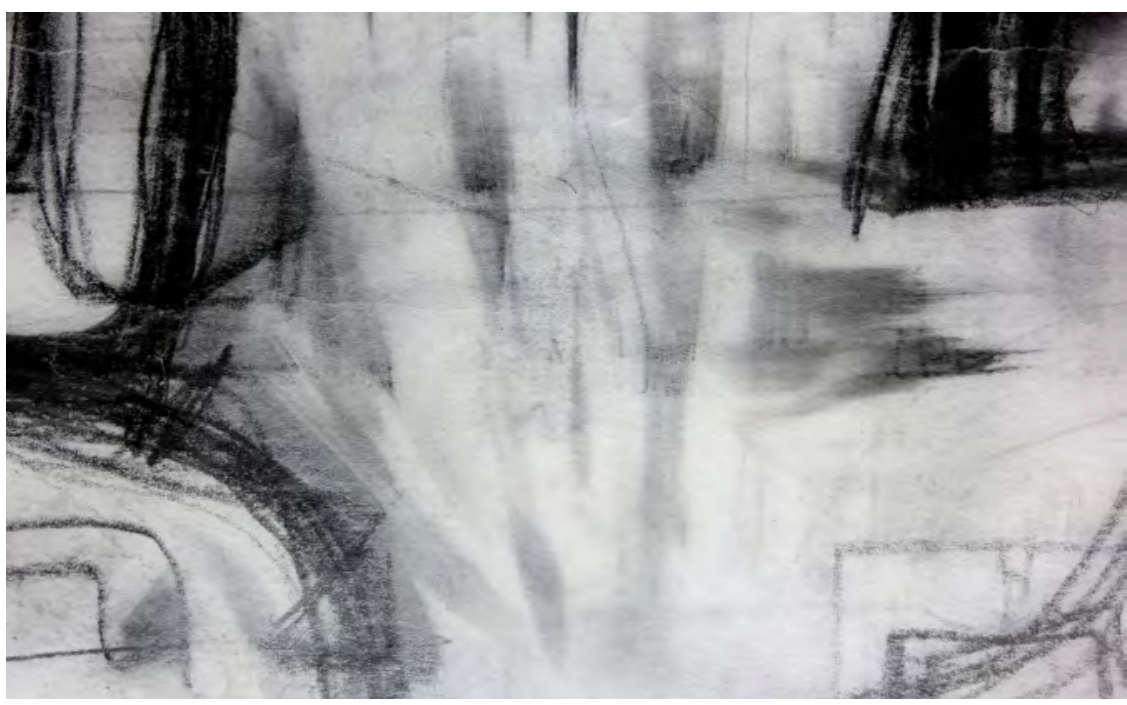

Fig. 2 Simon Twose (2018). Kaikōura canyon sea surface. [Video still]

Fig. 3 Simon Twose, Jules Moloney, Lawrence Harvey (2018) Canyon installation, Palazzo Bembo, XVI Venice Biennale [3D VR capture. [Photograph, Anastasia Globa] the canyon's powerful yet un-representable presence. The hybrid drawings are spatialised as an abstract virtual and aural sketch environment, where presences in the Kaikoura canyon and those in drawing are merged. The Canyon installation brings this spatial experience to a gallery situation. The installation is the first output of the Canyon research and was exhibited at Palazzo Bembo in the XVI Venice Biennale, 2018. The Canyon installation adds a sculptural and material component to the drawing research. It is conceptually an inhabitable sketch that projects the participant into the abstract world of the Kaikoura canyon while, at the same time, immersing them in the smudged dynamics of its drawing (Fig. 3).

Canyon is a collaboration between Simon Twose, Jules Moloney and Lawrence Harvey, researching open possibilities in architectural drawing. This paper reports on the first stage of the research and uses the Canyon installation as an armature for a brief discussion of findings. It observes that architectural drawing's relation to its subject matter is more than simply a scalar projection of a mark towards the space it represents, such as a line describing the contours of a landscape drawing is a site where multiple presences, in landscape and drawing, influence and inflect one another. This is most intense in the rapidly sketched

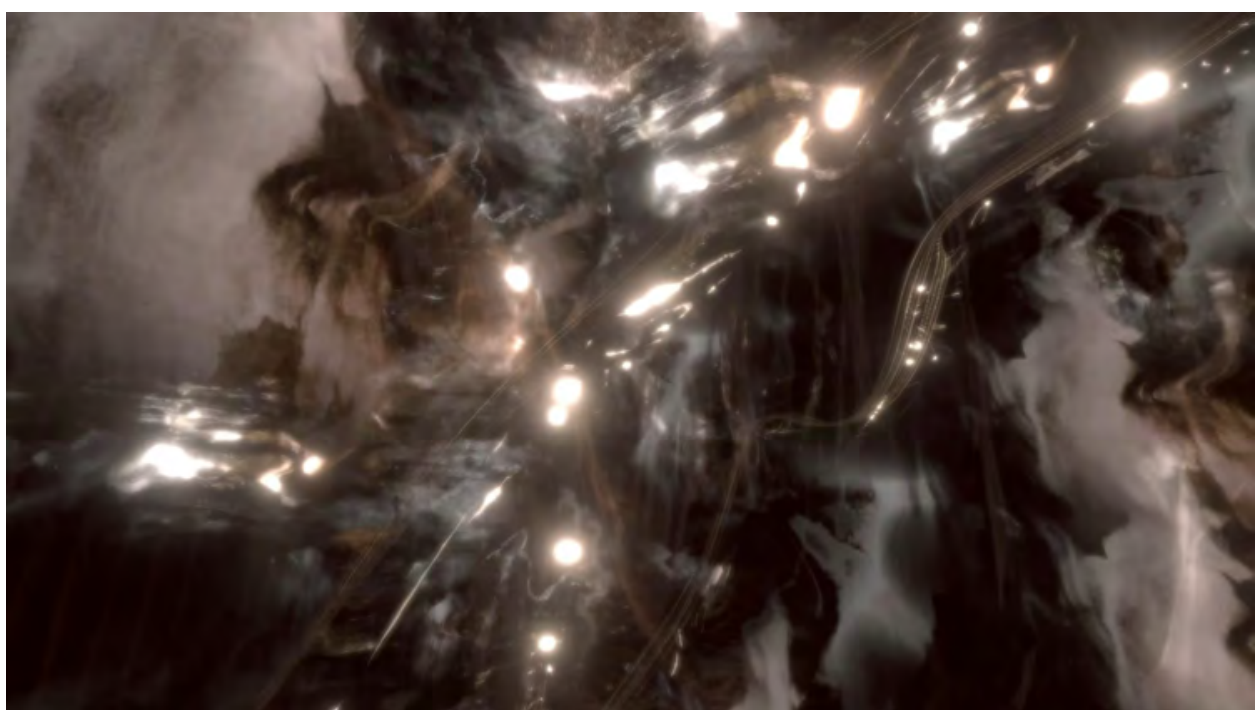




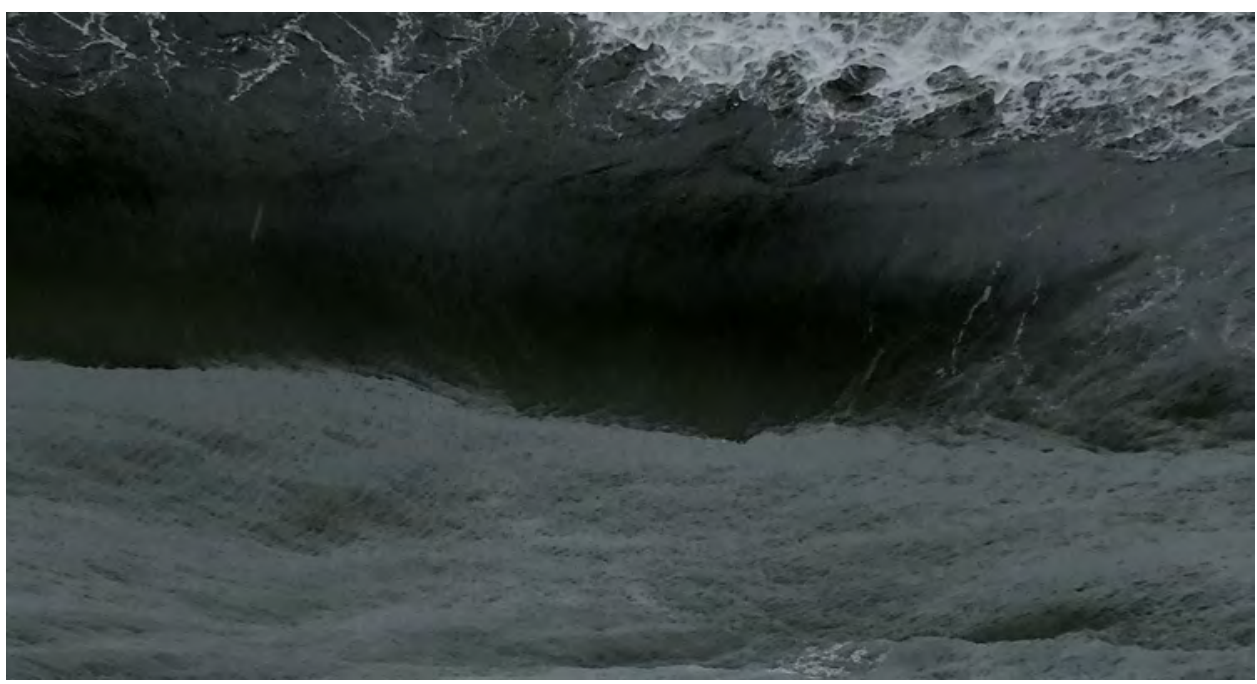

Fig. 4 Simon Twose, Jules Moloney (2018). Recalcitrant smudges in spatialized VR sketch environment [VR still Image, Anastasia Globa] smudges and "recalcitrant marks" of a spatial sketch. In this, the non-semiotic blurs take on a dual role. Their material performance combines with the material performativity of the spatial subject matter to evoke presences in both; the abstract blurs allude to physical and intangible conditions-of pressures or turbid flows, and imagined latencies. The sketch becomes a dynamic space of complex presences in sfumato relation. In sfumato, a painting technique perfected by Leonardo da Vinci, the subtle graduation of lights and darks creates a perceptual vibration between elements central to the gaze and shaded contours peripheral to it. It creates, in Vasari's words, a "hovering between the seen and unseen" (cited in Gombrich, 2004 [1959]: 185). The term is used in this paper to bring to mind a state of liminality, shifting possibility and asymptotic mutuality, in the relation between presences in drawing and in landscape (Fig. 4).

\section{Presence sketches}

A rapidly sketched section through the Kaikōura canyon picks up its general condition-a vertiginous drop in seabed in relation to a sea level datum (Fig. 5). But the sketch is also an action of the hand which inevitably has smudges and erasures. Its instrumental logic, as a section, merges with elements that are a-logical-unintentional marks that point to movement and flux of material (Fig. 6). The lines extend beyond their intended contour, they smudge and skip over the texture of what they are drawn on. The blurs and smudges start to talk of spatial and material conditions, of atmospheres, pressures, flows of rock and mud, and latent seismic movement; eruptions of unseen gasses and turbulence. Sketches like this are a way of observing intangible architectural conditions. Smudges and other "recalcitrant marks", as described by James Elkins, coalesce with affective, non-representational registers (1995). They prompt thoughts about atmospheres, flows and latencies. They are marks that record the "nuanced misalignments, approximate thoughts and imperfect moments [that...] resist fixing normative figuration" (Kulper, 2013: 63). 

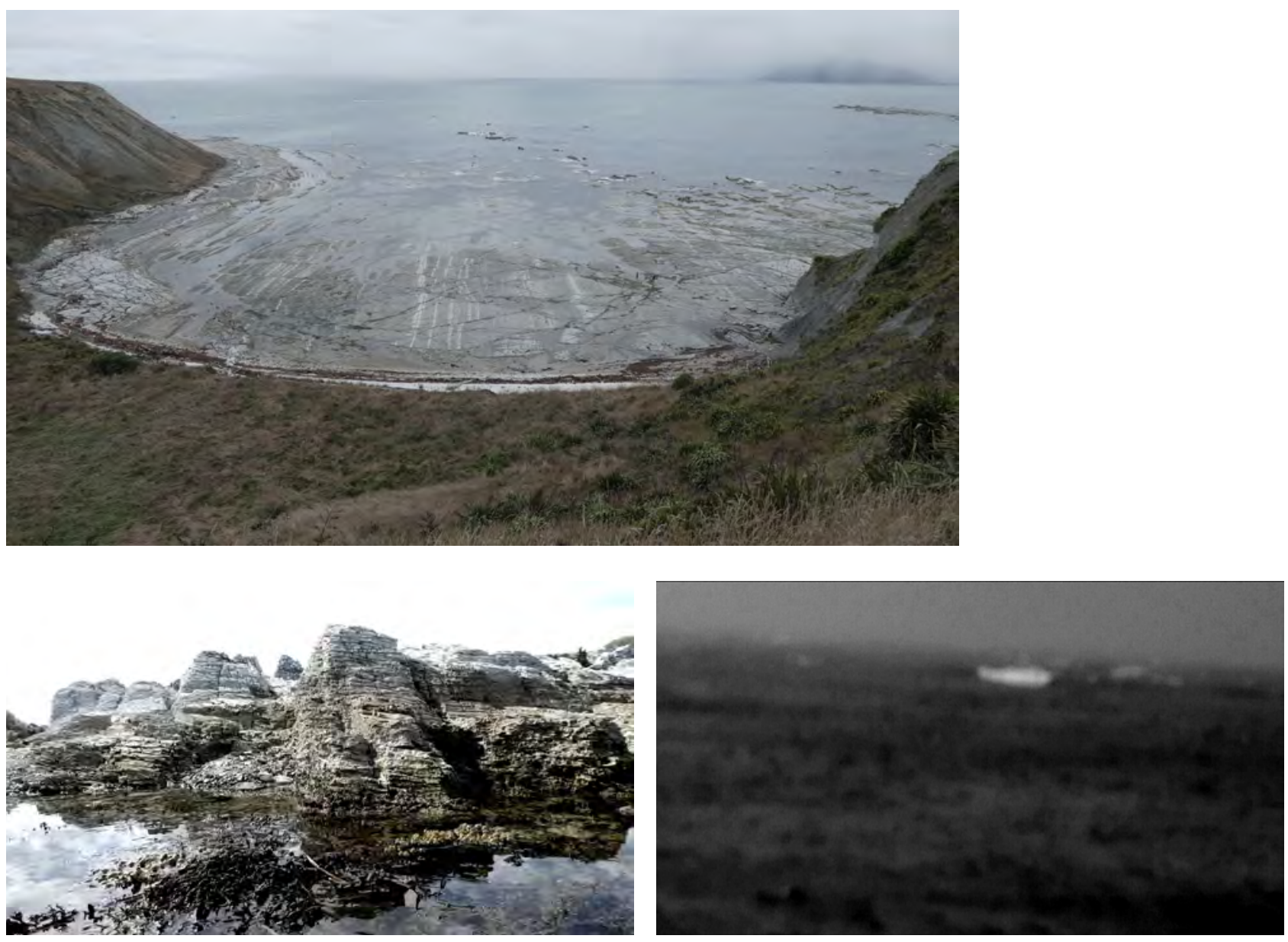

Fig. 5 Simon Twose (2018). Kaikōura Canyon section [Graphite on paper] Fig. 6 Simon Twose (2018). Kaikōura Canyon section [Graphite on paper]

Fig. 7a, 7b Simon Twose (2018). Rock surface sketch study [Photograph]

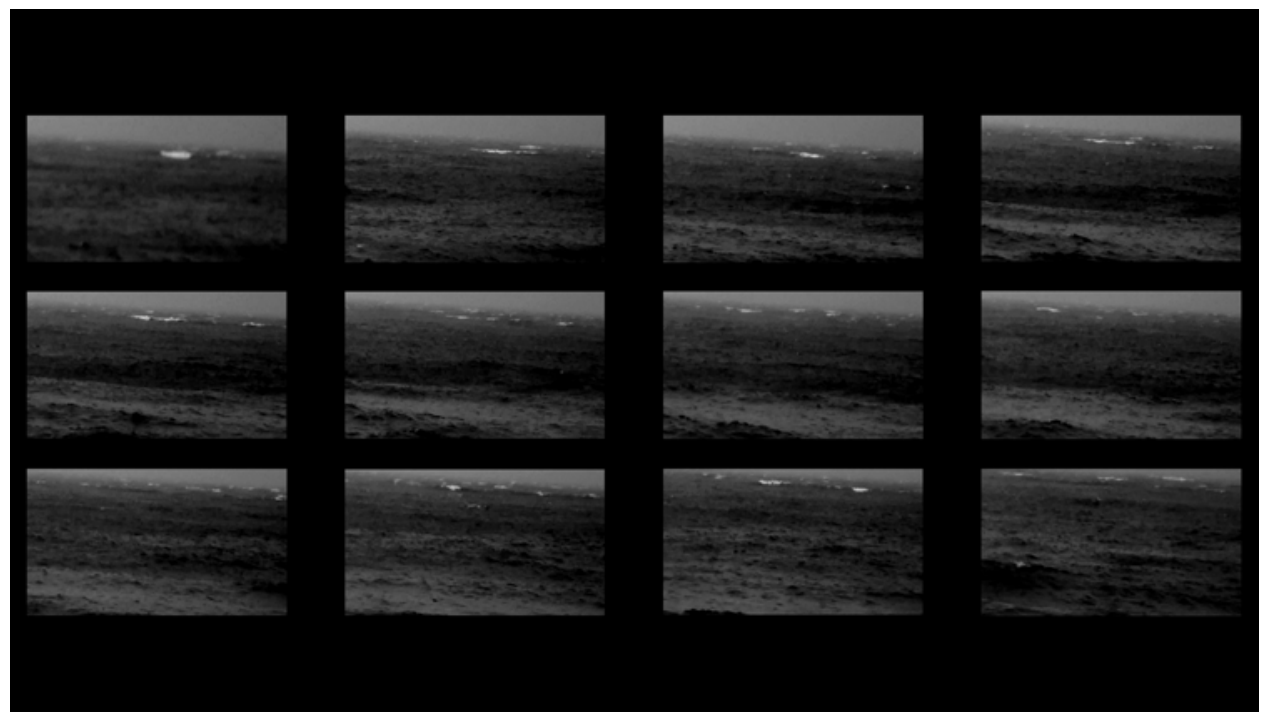

The Canyon analogue sketches were made by drawing sections, plans and three dimensional "scenes" over a rock-like surface. We made the graphite skip over the paper by the jagged shape of the rock underneath (Fig. 7). This skipping allowed unexpected elements to influence the drawing's marks. This was an exaggeration of the feedback normally found in analogue sketching and we used it as an analogue of the material dynamics of the canyon. The rock beneath the 
paper caused the marks to smudge and change direction, so that a sectional drawing of the sea floor became not a single line, but an indeterminate series of marks mapping the imagined presence of flows, pressures, mud and rock. Some of the lines are singular and fine, and describe pure boundaries, such as at the water's surface. Others describe transitions between materials that are less defined, such as at the muddy junction of sea and sediment-laden seabed, or where sea cliffs drop vertiginously into an imagined darkness. The sketches traverse the imagined space of the canyon, allowing scale and material to be amorphous in order to distil something beyond instrumental description (Figs. 8, 9).

The research focusses on the traces and marks that contribute to openness in drawing: the blurs, erasures and pentimenti in an architectural sketch-the redrawing of lines, one over the other, to strengthen one's thinking (Fig. 10). Pentimenti is used in art historical descriptions of art practice, and is useful in evoking the delicate dance between certainty and uncertainty in making drawings. Joan Faust, in discussing Leonardo da Vinci's drawings, talks of how, evidenced by "a welter of pentimenti", he engaged uncertainty in sketching:

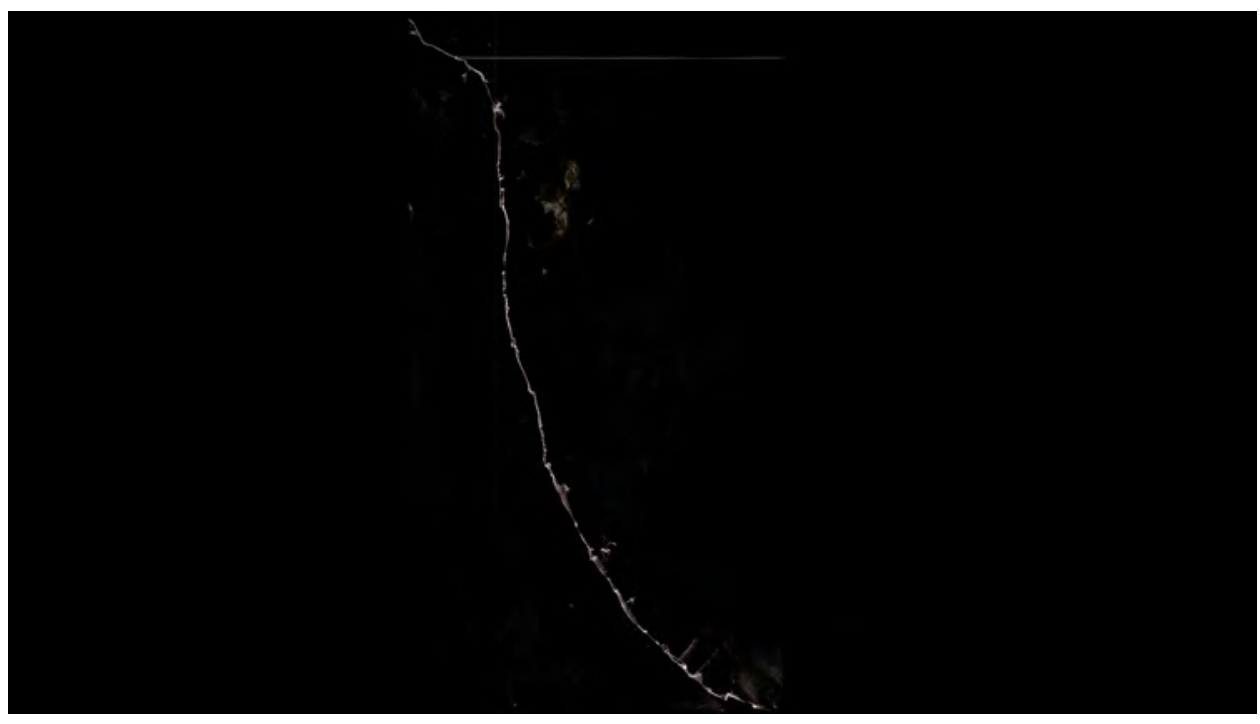

Fig. 8 Simon Twose (2018). Presencedrawing studies (details). Sediment turbidity. [Graphite on paper]

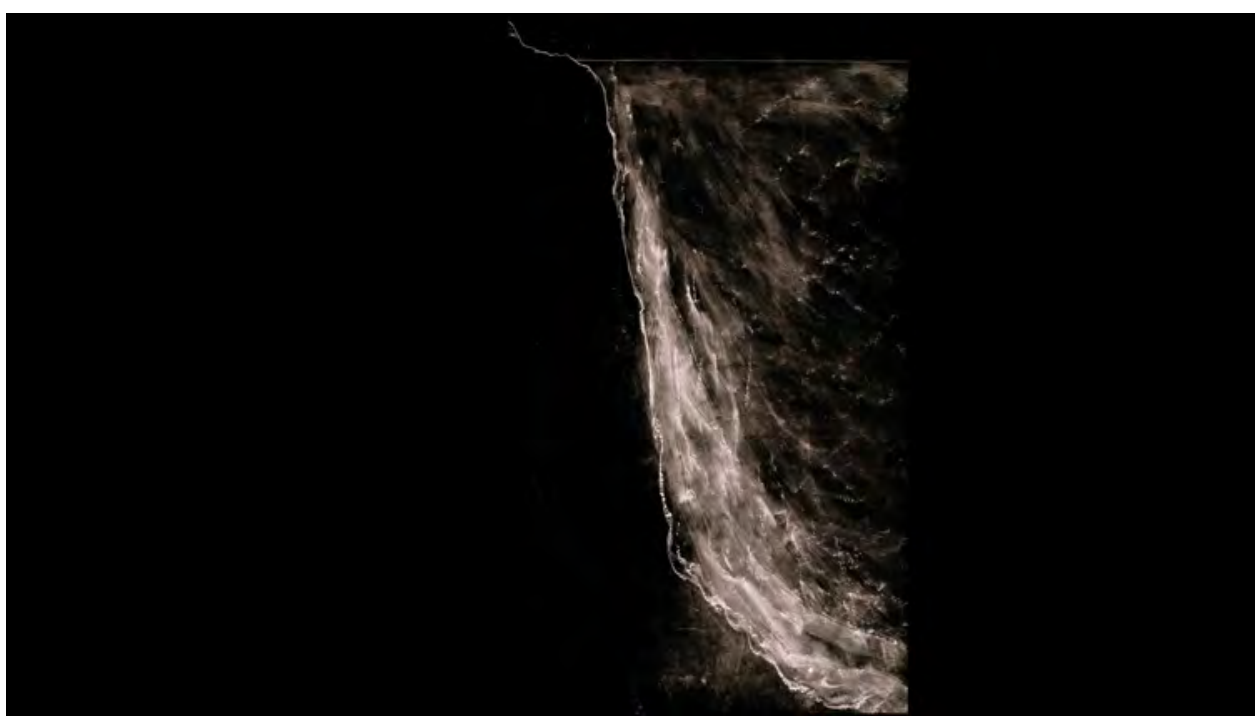



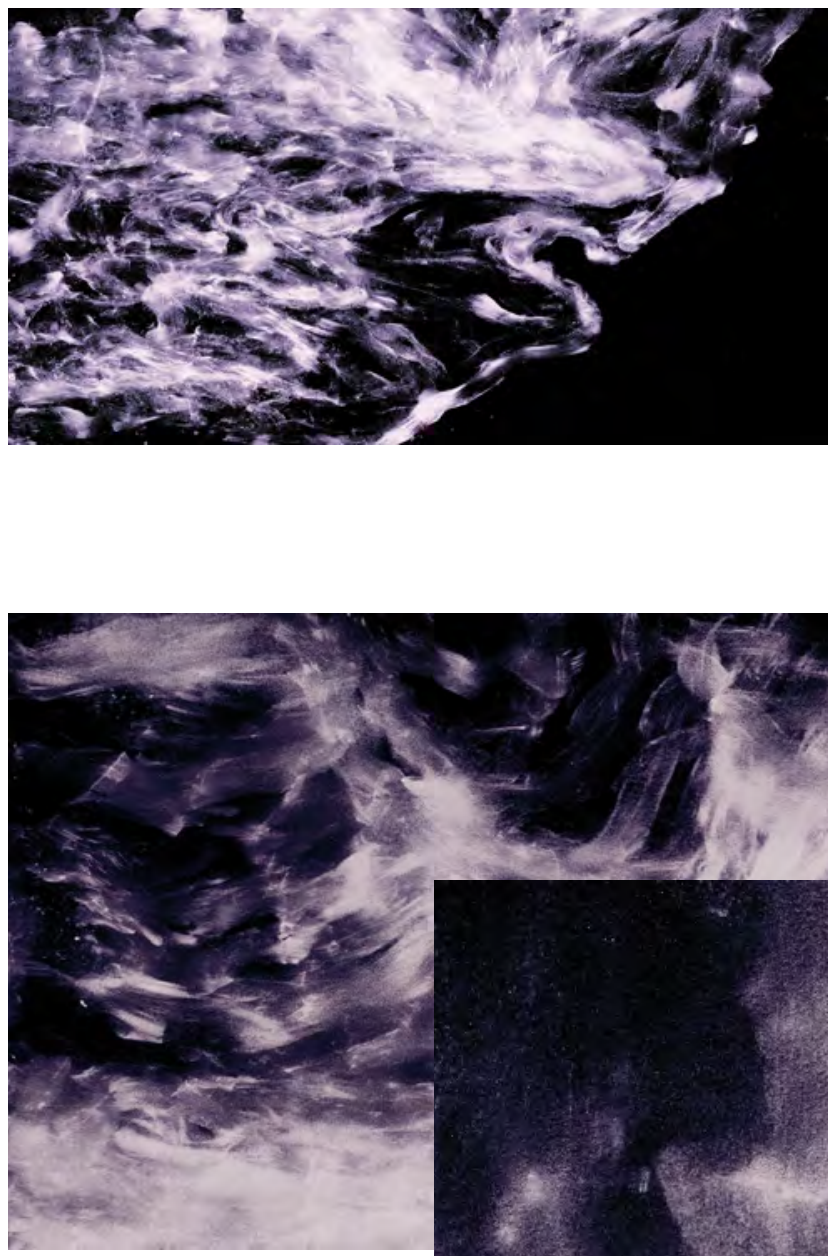

Fig. 9 Simon Twose (2018). Presencedrawing studies (details). Gas eruptions. [Graphite on paper]

Fig. 10 Simon Twose (2000). Recalcitrant smudges, blurs, erasure and pentimenti of the sketch. [Graphite sketch on butter paper]
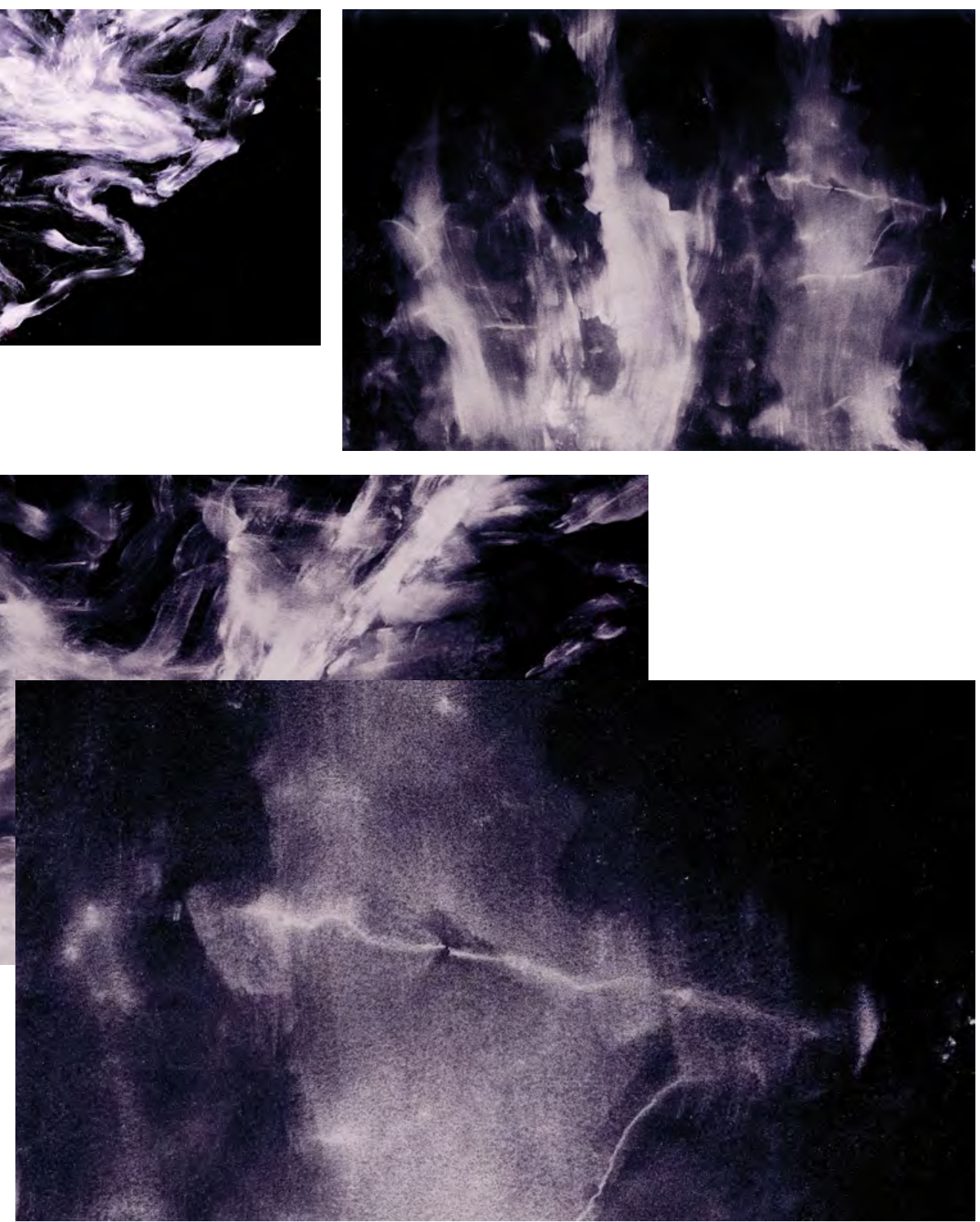

Leonardo conceived of the sketch as a liminal stage, a stimulation of the mind to further investigations not a preparation for a particular work. In his concept drawing becomes "a process which is constantly going on in the artist's mind," and instead of fixing the flow of imagination, the uncertainty of line keeps it in flux. (Faust, 2012: 81)

These irresolute marks contribute to spatial understanding, they are part of drawing as an active way of knowing, a "knowing immanent in doing" (Downton, 2003: 98). The indeterminate, blurred marks are not descriptive in an instrumental sense-but allude to abstract spatial qualities, such as immense scale, atmosphere or even the weight of material. They contribute to an understanding of presences, both within drawing and within drawing's subject matter. A sketch, such as a section, captures form and contour but is also inflected by what that space is imagined to be like; the sketch triggers images in the drawer's mind of pressure, weight, viscosity and unpredictable flows. An instrumental 
understanding mixes with one cultivated through spatial imagination-dark, heavily drawn lines align with the obdurate mass of rock, fine curved lines with motile flows and pressures.

Gestural analogue drawing has traditionally been associated with intangible, qualitative dimensions. Sketches are open: evocative, indeterminate and unfinished, and therefore, full of possibility. Drawing is, to paraphrase Nancy, nascent, "the opening of form" (2013: 1); it is necessarily irresolute. To Elkins the "recalcitrant, 'meaningless' smears and blotches" (1995: 860) that surround the interpretable line hover between logic and a-logic. He calls them non-semiotic marks, with the power to evade interpretation, "shimmering thing(s) at the edge of analysis" (858). This aligns them with intangible spatial understandings, such as flows, atmosphere or presence. In this way, a smudged non-semiotic mark, made in concert with architectural imagination, is an uncertain mode of inquiry that embraces aleatory, agrammatical ways of knowing, to use Sarat Maharaj's terms, engaging modes that are "beyond the organizing, classifying spirit of grammar" (2009: 4). Maharaj makes the case for these uncertain aspects in art practice to be key to it as a complex and unique way of knowing. Rather than the "methodological steel tracks" of know-how, it is a pursuit of no-how (3). It is part of a cultivation of non-method necessary to maintaining uncertainty, which Maharaj argues is crucial to creative practices. A non-method where the openness of creative production prompts the discovery of intangible conditions, such as presences.

The Canyon project pursues sketchy indeterminacy into other media. It tests how analogue and digital media, and spatialized sound, can intensify the "tensions and intensities, and pentimenti, of mark making” (Clift in Nancy, 2013: back cover). And consequently, how an intensification of these open, abstract conditions might radicalise drawing's projective connection to space beyond it, exploring it as a space of sfumato presences, in drawing and landscape.

\section{VR sketch-space}

In the Canyon VR, we made the blurs and pentimenti of sketched marks three-dimensional and bodily navigable; a participant in the virtual environment is within a weather system of lines, smudges and grainy marks that are at huge scale and constantly changing in form and density. In this virtual sketch environment, the a-logical, non-semiotic marks, which allude to shared presences in the Kaikoura canyon and in the material performance of the sketch become an endlessly transforming substance. They form an unbounded sketch-space that is canyon-like in its scale and an abstract evocation of flows, pressures and seismic jolts. Viewers within the VR sketch-space are swept along by currents and navigate within a world of irresolute marks with no reference to their location or scale: marks appear huge, and pass, like ocean swells, or spawn endlessly in the distance creating a dynamic space with no discernible boundary. The subject of the sketch, the sea, becomes entangled with the spatiality of the digital medium. Presences in drawing and landscape are palpable through the kinematics of VR (Fig. 11).

In Canyon, VR is a sketchy medium, similar to the traditional architectural sketch. We configured the virtual environment through four types of mark, extracted from the analogue drawings: contour, flow, space and smudge. Each 


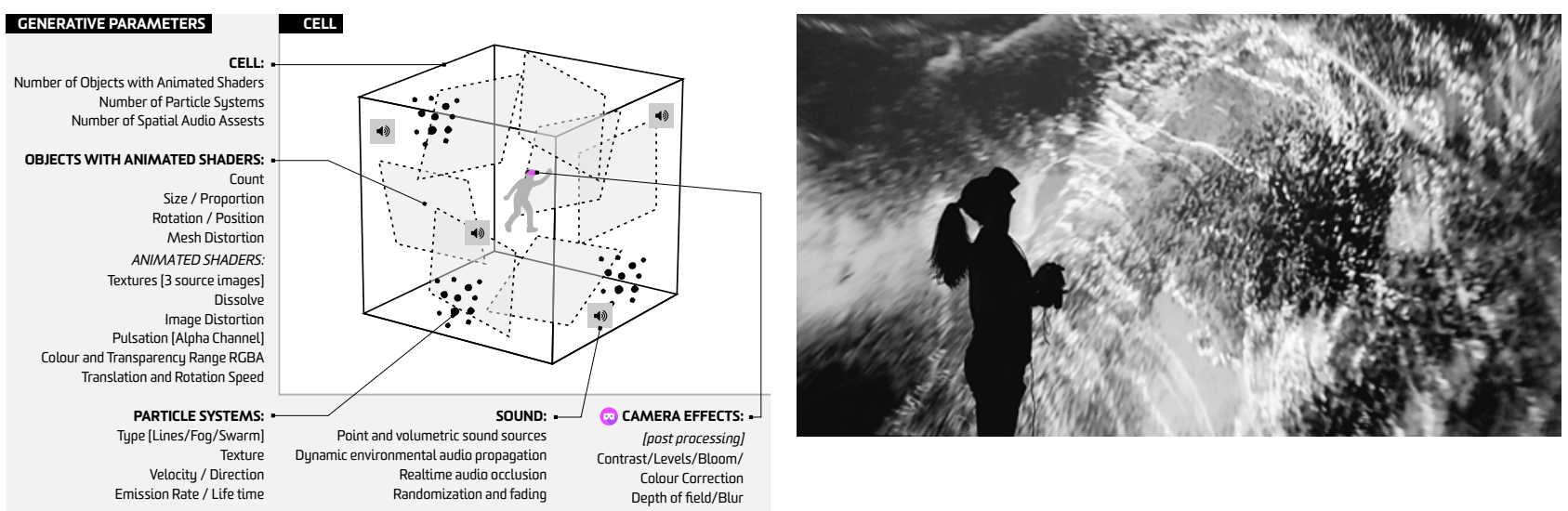

Fig. 11. Jules Moloney (2018). Canyon installation, viewer in VR sketch environment. [VR still Image, Anastasia Globa] mark is then placed in flux via the capacity of the software to interactively adjust graphic variables such as transparency, colour, scale and granularity. Further transformation occurs autonomously via a spawning system, in which a 'parent' mark spawns a 'child' that has independent behaviours and spawns further, independently behaving child objects. These propagating families of marks oscillate within an environmental milieu of dynamic particle systems and lighting effects, and are experienced via an array of camera manipulations.

Our strategy of exploiting the technical affordance of VR, to extend drawing's traditional openness, contrasts with typical approaches to VR that are primarily concerned with verisimilitude. The reference point for architects using VR is the use of animation, which as documented by As and Schodek (2008) has been developed primarily to communicate the kinematic experience of architecture. Traditions of projective geometry condition the practice of animation that underpins spatial representation and the technical apparatus of the animation camera, which utilises principles of perspective. In the Canyon project this is radically shifted. We reduced perspectival representation of 'real' space in favour of the space of an open, sketch-like indeterminacy; rather than passively presenting the viewer with a virtual version of reality, VR provides an environment available for multiple interpretations, as in an open sketch. This raises questions about how the immersive kinematics of VR can be a creative drawing medium, effectively intensifying the traditional openness of a sketch. We are interested in the architecture that this three-dimensional sketch environment provokes, and how the immersive medium of VR can afford conceptual openness, how it might distil things beyond instrumental description, such as presences.

\section{Sonic sketches}

A landscape of blurred and smudged sounds accompanies the navigation of a viewer, or drawer, in the Canyon VR space and installation. Sonic sketches that blur and smudge and jolt their experience (Fig. 12) confront participants moving through here. Lawrence Harvey created six fluidly interconnected soundscapes to correspond to aspects of the presence sketches. These have qualities of granularity, pointillism and unexpected shifts in scale and intensity gleaned from the sketches and the canyon subject matter. Harvey notes that the temporal organisation for the sound design can be likened to a mobile slowly turning in the room where the sonic layers are circling or revolving within each other at different 


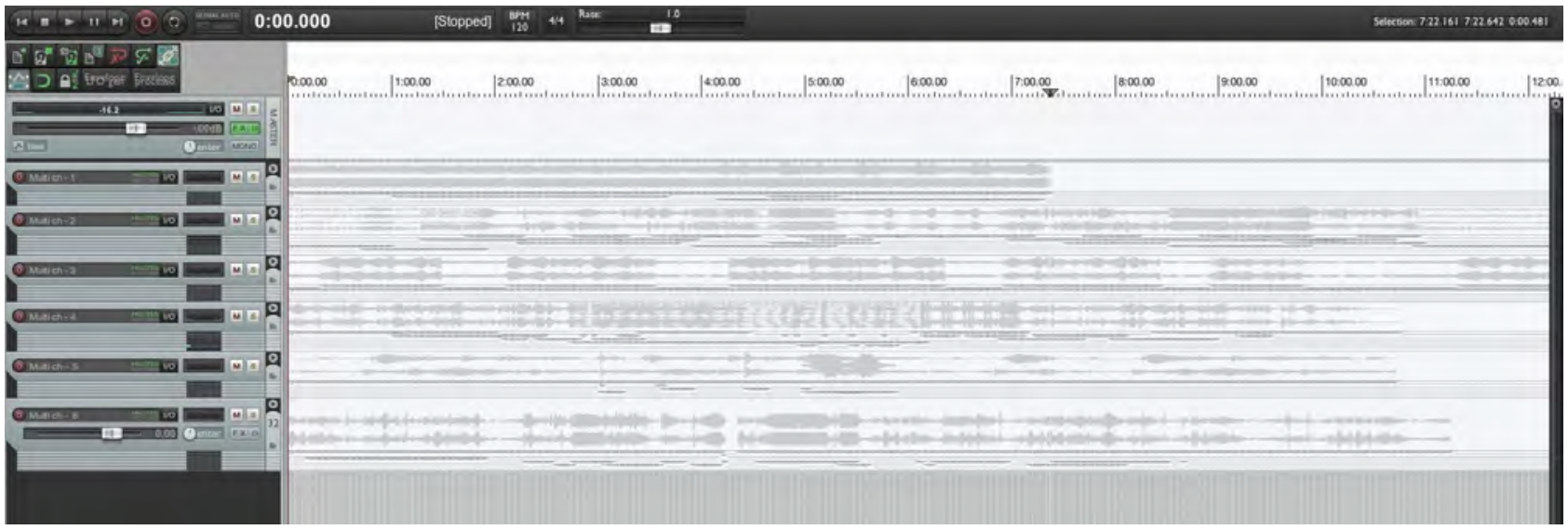

Fig. 12 Lawrence Harvey (2018). Canyon installation soundscape design. [Screen shots of Reaper program. Screenshot, Lawrence Harvey]

Fig. 13 Simon Twose, Jules Moloney, Lawrence Harvey (2018). Canyon installation, Palazzo Bembo, XVI Venice Biennale [Photograph, Anastasia Globa] cycles. The soundscapes are composed to accentuate the motion inherent in the sound, and to provide a sense of fluctuation in the environment; the sound intensifies the experience of textural motion and flows, to "connect the tar paper folds, dents, cavities with an unsettled continuum of forces" (Twose, Moloney, Harvey, 2018: 189). In the canyon project, sound is a way of drawing, as a spatial and sensorial way of sketching the Kaikoura canyon landscape. It augments the appreciation of the sketch, taking its evocative aspects and making them appreciable in a non-visual, spatial and sensorial way. In this sense, the sonic drawings delve into the spatiality of the sketch, distilling aspects that evade instrumental description, aspects that are bodily appreciable, and not easily interpreted as representational marks.

\section{Canyon installation at XVII Venice Biennale}

Canyon was a mixed-media installation. It was a dark, immersive space formed by a forty metre drawing in black tar-paper, crumpled to form an enclosed space within the gallery. Six overlapping soundscapes within the space accentuate the creases and spatial dislocations created by the tar-paper drawing. Four small video screens were visible through fissures in the drawing. These allowed glimpses into a virtual environment, composed of continuously morphing, abstracted sketches. These flickering images provided the only light in the space, and they occasionally flashed brightly, jolting the space in a similar way to the soundscapes. Participants entering the dark drawing space experienced a gradual dislocation from "normal" architectural space as their eyes adjust to the darkness. They entered into an abstract world of creased black surface, shifting soundscapes and digitally generated sketches (Fig. 13). Once in the small space of Canyon, viewers were conceptually within an inhabitable drawing. They were within both a single mark and an unbounded, abstract submarine landscape. Prompted to move around the dark space, following the crumpled surface and soundscapes, visitors discovered the flickering sources of light from the small VR screens showing through fissures in the surfaces. 


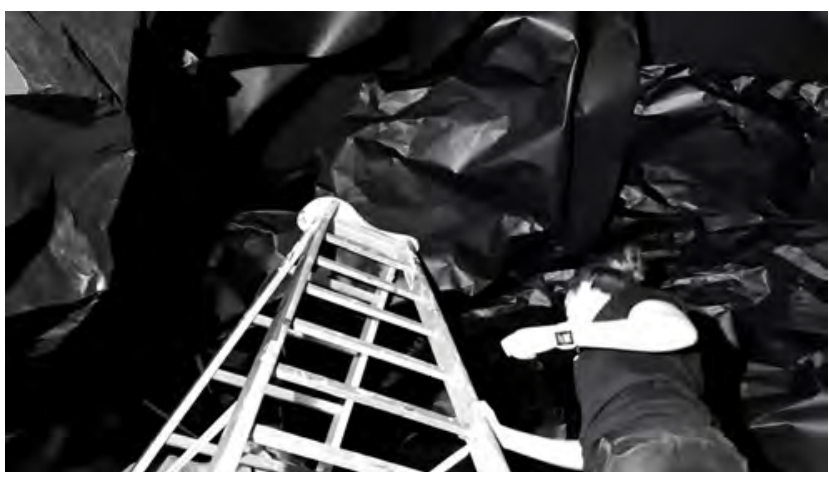

Fig. 14 Simon Twose, Jules Moloney, Lawrence Harvey (2018). Canyon installation being physically drawn. [Photograph, Anastasia Globa].

Fig. 15 Simon Twose, Jules Moloney, Lawrence Harvey (2018). Canyon installation, vertical panorama recording. [Photograph, Simon Twose].

Fig. 16 Simon Twose, Jules Moloney (2018). Canyon VR environment: VR navigation of installation space and presence-drawings. [Video still, Anastasia Globa].

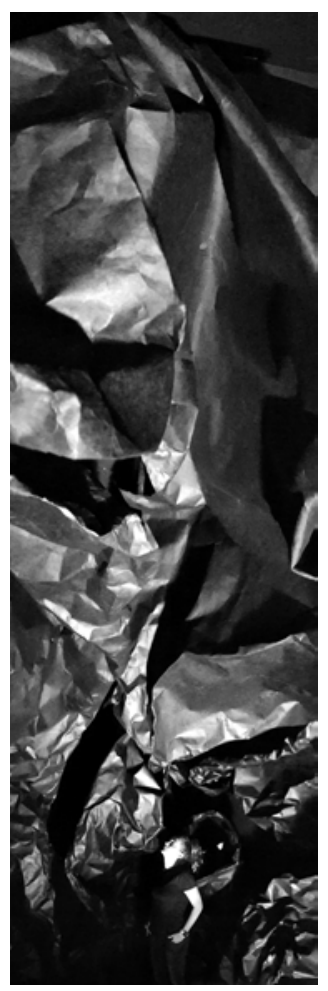

The black forty metre drawing was 'sketched' in the space by deforming the paper in response to intensities, pressures and flows imagined in the canyon (Fig. 14). In this sense, it was an extension of ideas pursued in the canyon research, extending the gestural potential of drawing in response to a material feedback, as a way of coalescing two asymptotically related presences-in landscape and drawing. The tar-paper sketch was in response to the spatiality emerging from the VR and sound sketch-spaces, experimenting with how these might work as a spatially appreciable, sculptural space. The installation was in effect a fourth aspect to the hybrid drawing, augmenting the hand drawn, VR and sonic drawing modes.

\section{The room poses a question}

The installation was very difficult to record; it was very dark and immersive so relied on sensory experience. It was also unlike a traditional architectural exhibition. An expectation of seeing representations of architecture, when entering the space, quickly dissolved as one's eyes adjusted to the darkness and the soundscapes imposed themselves on the senses-and the space itself began bodily to pose a question. It had a strange atmosphere not recordable solely in visual images. We tried several ways to capture this strangeness. We took panoramas that unravelled the crumpled drawing, captured the space in 3D imagery and took 3D videos of our interacting with the space. We put these into a VR environment, which had the odd effect of allowing one to inhabit the installation with one's previous self (Figs. 15, 16). We also recorded the drawing in material, with imprints of the creased surface cast in concrete and wax (Figs. 17, 18).

These recordings, in images, video and castings, are similar to the pentimenti of sketches-the many lines drawn over one another in the same place. They are part of an ongoing process of sketching that is about distilling presences, bringing them into space and material while keeping them sketch-like and irresolute. Each mode of recording opens another set of possibilities and corresponding non-discursive registers, so is part of an iterative progression, each is a mark standing in for something beyond it.

The acts of making involved in this ongoing process of sketching, recording then re-sketching, raise the question of drawing's poiêtics. Poiēsis (Ancient Greek: $\pi \circ i \eta \sigma \iota s)$ is the etymological root of poetics and is related to techne, or the making of things; the formative, becoming of form. In Heidegger's words, "techne is 


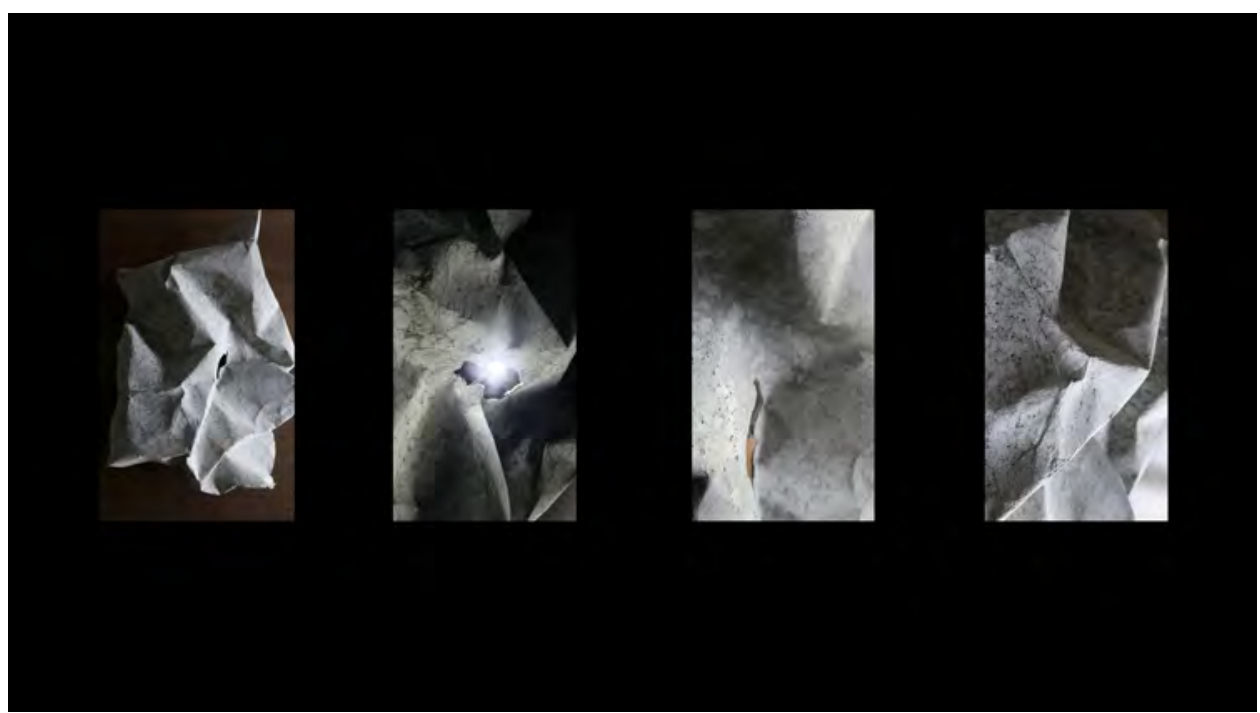

Fig. 17 Simon Twose (2018). Canyon installation recording, concrete imprints of the drawing surface. [Photograph, Simon Twose]

Fig. 18 Simon Twose (2018). Canyon installation recording, wax/ tar-paper surface imprints. [Photograph: Simon Twose]

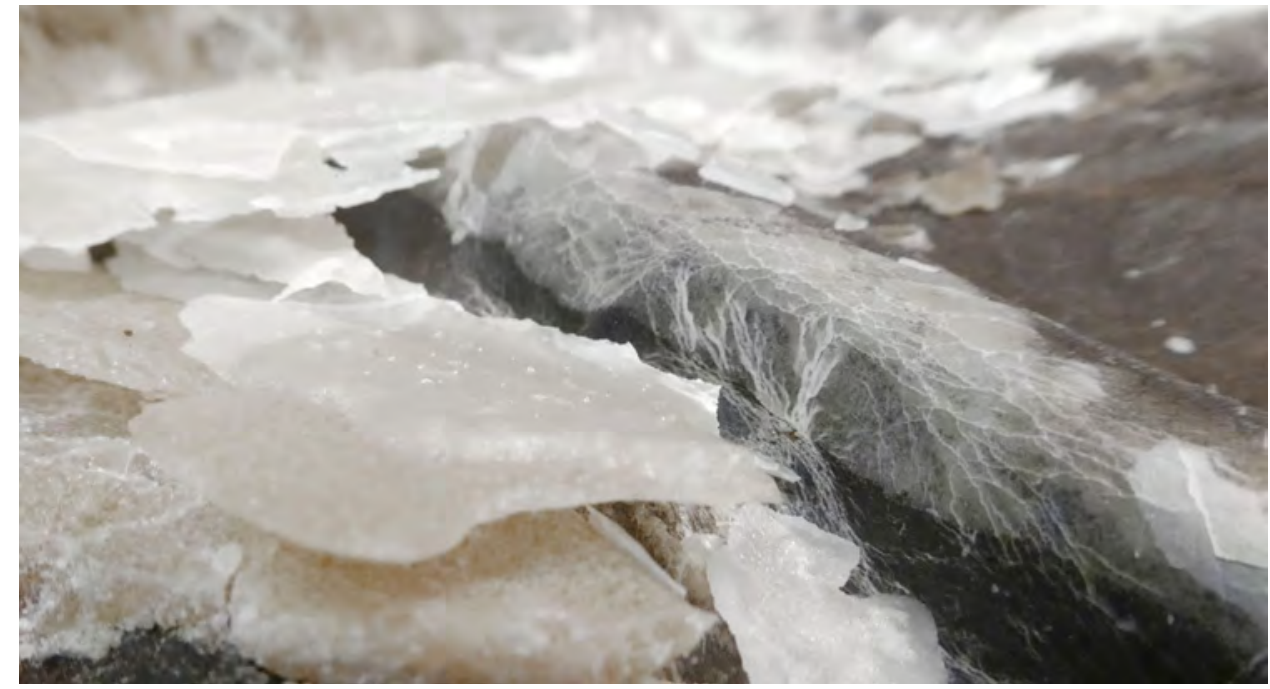

the name not only for the activities and skills of the craftsman, but also for the arts of the mind and the fine arts. Techne belongs to bringing-forth, to poiēsis; it is something poietic" (1977: 5). Poiêtic marks stand in for something beyond them, they are irresolute and in formation, referring to further possibilities. This is close to the way architectural drawings operate, they are an archive of making, a sea of marks that stand in for, and bring forth, space outside them, such as a building or landscape. The acts of making in an architectural drawing, such as in the full size sketching of the tar paper, acknowledge the poietics of an architectural mark, that is, constantly in a state of referring outside itself while responding to the non-discursive characteristics of its making. Poiessis is part of the emergent, perpetual bringing forth quality of a sketch, capturing complex qualities such as the "invisible extremity of the mark [trait], the point whereby the line advances and loses itself beyond itself in its own desire" (Nancy, 2013: xiii). The focus on the shared space of drawing's making and the performative agencies of landscape opens the possibility of a poietic architecture; a space of transference where drawing and landscape are in continual emergence or nascence. The Canyon hybrid drawings, and installation, are concerned with distilling this poiettic spatiality. 


\section{Sfumato presences}

We use the term sfumato as a metaphor of relation. It is a way of imagining the difficult connection between drawing and space beyond it. Sfumato is the active crossing and blurring of one thing with another, taking care "that your shadows and lights be united without strokes or marks, in the manner of smoke" ("senza tratti o segni, a uso di fumo") (Leonardo quoted in Nagel, 1993: 11). It is a useful metaphor to evoke a complex intra-action of things, such as light and dark, drawing and building, drawing and landscape.

Sfumato alludes to a tending towards but never complete intersection of one thing and another. It aligns with sketching's poiēsis, of a mark in the process of being made and becoming, or referring to, something beyond it. We use the term to evoke a hovering of presences, between drawing and the space it draws; it has a sense of open possibility and cloudy movement. Sfumato is a metaphor that suits the Canyon research's ambition to problematise connections between things, such as the ideas and phenomena in architectural drawing, and it also paints an atmospheric image, of smoky indeterminacy, that closely aligns with the strange spatiality that appeared in the Canyon VR, soundscapes and installation.

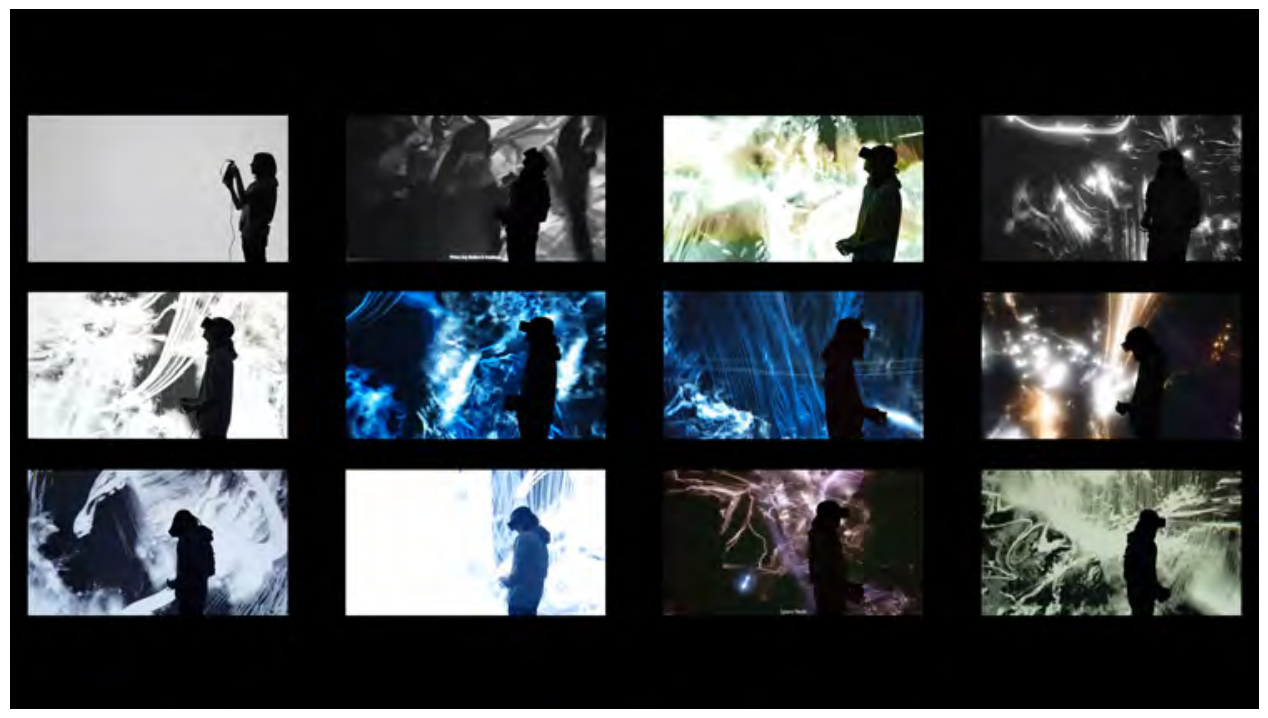

Fig. 20. Simon Twose, Jules Moloney (2018). Canyon VR environment: smudge zone of VR environment. [Video still, Anastasia Globa].

\section{Future work}

At present Canyon is in the mode of observational architectural drawing. Drawings are in a sense archives of thinking, by way of many acts of marking; they are fields of collected impressions. These impressions are tied to the space in which the marks are made, as much as the ideas and subject matter they respond to. As architect Riet Eekhout says, drawing is a way of "speculating on the nature of a subject or object ... and how, through drawing, their presence can be activated" (2014: 9). It is a way of extending sensory capacity, towards the discovery of things that might hover between the seen and unseen, revealing the "appearance of what was never hidden" (Nancy, 2013: 105). Drawing is also generative, it is a way of inventing space and form. The next stage of Canyon will continue to develop how presences, distilled through hybrid drawing, play a role in ideation; how the shared space of drawing and landscape generate architectural space and form, while maintaining a nascent, sketch-like ever-emergence. Canyon is part 
of an ongoing project in open architecture, an attempt to sketch an architecture that hovers between drawing and landscape, composed of presences hovering in sfumato relation.

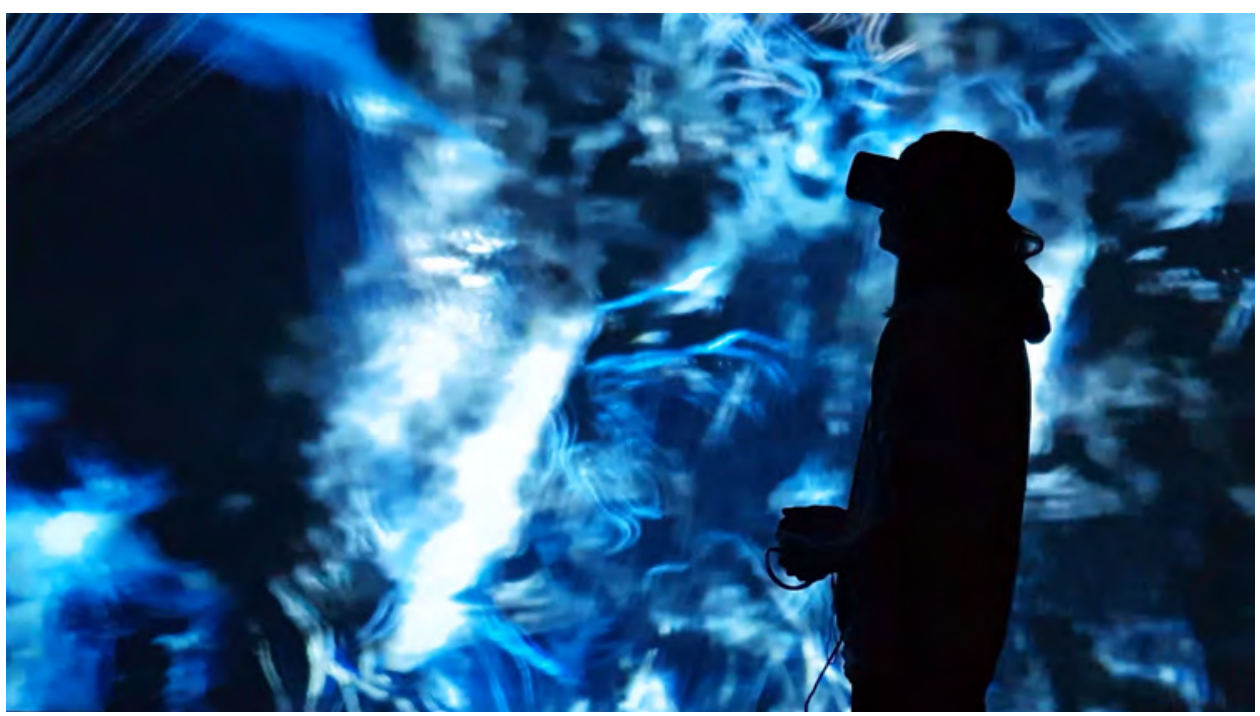

REFERENCES

As, I., Schodek, D. (2008).

Dynamic digital representation in architecture: Visions in motion, London, UK: Taylor and Francis.

Chard, N., Kulper, P. (2013). Pamphlet architecture 34:

Fathoming the unfathomable.

New York: Princeton Architectural Press.

Downton, P. (2003). Design research. Melbourne: RMIT University Press.

Eeckhout, R. (2014). Process drawing (Unpublished doctoral thesis, RMIT University, Melbourne, Australia).

Elkins, J. (1995). Marks, traces, "traits", contours, "orli", and "splendores": Nonsemiotic elements in pictures. Critical Inquiry, Vol. 21, No. 4, pp. 822-860.

Faust, J. (2012). Andrew Marvell's liminal lyrics: The space between. Newark, DE: University of Delaware Press.

Gombrich, E. H. $(1959,2004)$. Art and illusion: A study in the psychology of pictorial representation, New York, NY: Phaidon Press.
Heidegger, M. (1977). The

question concerning technology (W. Lovitt, Trans.). New York, NY: Harper \& Row.

Nagel, A. (1993). Leonardo and

Sfumato. Anthropology and aesthetics, Res 24, pp. 7-20.

Nancy, J-L. (2013). The pleasure in drawing (P. Armstrong, Trans.). New York, NY: Fordham University Press.

Maharaj, S. (2009). Know-how and no-how, stopgap notes on "method" in visual art as knowledge production. Art and Research, A Journal of Ideas Contexts and Methods, Vol. 2, No. 2, pp. 1-11.

Twose, S., Moloney, J., Harvey, L. (2018). Canyon: Experiments in drawing through analogue sketching, sound and virtual reality. In D. Maxwell (Ed.), Proceedings of 1st Annual Design Research Conference (ADR18) (pp. 179-194). Sydney: University of Sydney. 